\title{
Bad metals get ultra cold
}

\author{
Bad metallic transport in a cold atom Fermi-Hubbard system \\ Authors: Peter T. Brown, Debayan Mitra, Elmer Guardado-Sanchez, Reza \\ Nourafkan, Alexis Reymbaut, Simon Bergeron, A.-M. S. Tremblay, Jure Kokalj, \\ David A. Huse, Peter Schaus, and Waseem S. Bakr \\ arXiv:1802.09456
}

\section{Recommended with a Commentary by Ehud Altman, UC Berkeley}

Fermi liquid theory has been remarkably successful in describing transport properties of conventional metals. However the theory fails to explain the behavior of the resistance in a rather broad class of correlated materials, including in the normal state of the high $T_{c}$ cuprate superconductors. A striking violation of Fermi liquid theory in these materials is the linear dependence of the resistivity on the temperature over a broad regime, from the lowest measured temperatures up to the melting point near $1000 \mathrm{~K}$. This is in contrast to the quadratic temperature dependence $\rho \propto T^{2}$ that would be expected due to weak scattering of quasi-particles in the low temperature limit.

The behavior in the higher temperature regime is also hard to reconcile with any picture of quasi-particle transport. As long as quasi-particles exist, the resistivity is given by the Drude formula $\rho \sim\left(h / e^{2}\right) k_{F}^{2-d}\left(E_{F} \tau / \hbar\right)^{-1}$. Even if the linear temperature dependence was due to some unidentified temperature dependent scattering process within the quasi-particle picture, then this behavior should change drastically at the temperature where the quasiparticle lifetime becomes short compared to its natural oscillation frequency, i.e. where $E_{F} \tau \sim \hbar$. Hence, the Drude description should break down when the resistance reaches $\rho_{*} \sim\left(h / e^{2}\right) k_{F}^{2-d}$, known as the Mott-Ioffe-Regel (MIR) limit. Indeed the resistivity of most conventional conductors shows signs of saturation to a plateau close to $\rho_{*}$. In contrast, the linear dependence of the resistivity in the strongly correlated metals continues uninterrupted through the MIR limit, beyond which the quasi-particle picture cannot hold. This suggests that the behavior at low temperature, below the MIR limit, is also not a result of quasiparticle scattering. Metals showing such resistivity in excess of the MIR limit are commonly referred to as bad metals.

Over the years, a variety of theoretical models have been proposed to explain some of the above phenomenology, including marginal Fermi liquid theory [1], coupling to quantum critical fluctuations [2], and large-N models [3, 4]. Though these models lend useful insight, they lack a solid microscopic justification. In addition, because of the complexity of the materials showing bad metal behavior, it is hard to perform controlled experiments that would distinguish between different theories. The materials often exhibit many other effects, such as instabilities to various ordered phases, coupling to lattice degrees of freedom and 
disorder, which further complicate the task of separating the effect of purely electronic correlations.

In this context the work by Brown et. al., using ultra cold ${ }^{6} \mathrm{Li}$ atoms in an optical lattice, presents a breakthrough in the field. Not only were they able to observe bad metal behavior in a strongly interacting Fermi system, their approach holds several unique advantages in its ability to narrow down the possible underlying mechanisms. First, the microscopic hamiltonian, which governs the observed phenomena in the experiment, is well known and controlled. In fact the hamiltonian is precisely the fermionic Hubbard model on the square lattice

$$
H=-t \sum_{\langle i j\rangle, \sigma} c_{\sigma i}^{\dagger} c_{\sigma j}+\text { H.c. }+U \sum n_{i \uparrow} n_{i \downarrow},
$$

a well known minimal model for studying the physics of strongly correlated electrons. All parameters of the Hamiltonian were under control in the experiment, and the shape of the trap was engineered to give a flat potential leading to a constant filling around the center of the trap. The Data was obtained in the regime where the model is intrinsically strongly coupled $U \sim 8 t$ and close to half filling of the lattice $\langle n\rangle \approx 0.85$. Because there is neither disorder nor lattice phonons in this system, it ensures that all the observed effects are purely a result of the "electronic" correlations.

Second, the cold atom experiment allows to decompose the temperature dependence of the resistivity into distinct contributing factors and measure them in temperature regimes that are not accessible in solids. By making clever use of their state-of-theart imaging system, Brown et. al. where able to independently measure both the particle diffusion constant $D(T)$ and the compressibility $\chi(T)$ of the liquid. The diffusion constant, was measured by imposing weak density modulations of varying wavelengths on the system and measuring their decay times under the Hamiltonian evolution. The compressiblity was extracted from measuring the equilibrium density profile in the trap, which within a local density approximation gives $(\partial n / \partial \nu)$. finally, the conductivity was constructed from the compressibility and the diffusion constant using the Nernst-Einstein relation $\sigma(T)=\chi_{c} D$.

The natural regime for measuring conductivity in solids is the ultra-low temperature regime $T \ll E_{F}$. Ironically, such low relative temperatures are not yet achievable with ultra-cold atoms. Rather, the exper-

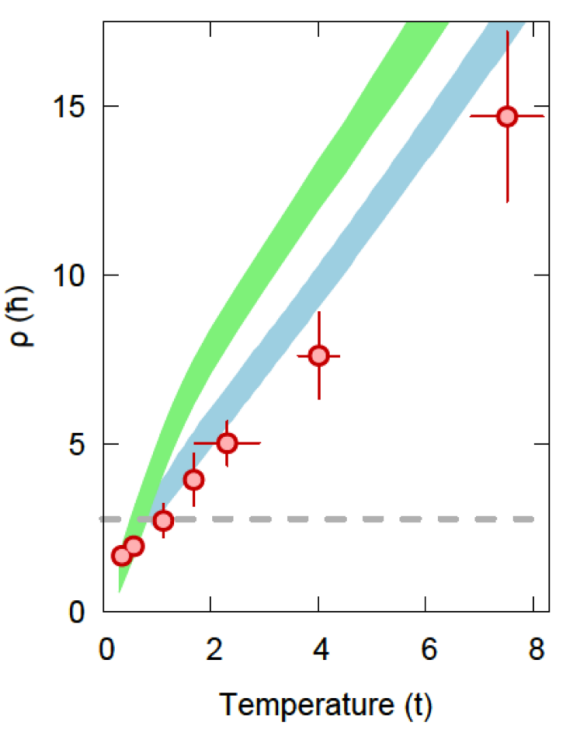

Figure 1: Resistivity as a function of the temperature as measured in the experiment (red circles) compared to exact diagonalization (blue band) and a DMFT calculation (green band). iment spans the range from moderately low relative temperature $T \approx 0.3 t$ to the high temperature regime $T \sim 8 t$. Thus the cold atom experiment is providing new information on the interpolation between two potentially simple regimes. At ultra-high temperatures $T \gg 8 t$, one expects $\rho \propto T$ simply because of the the 
universal high temperature limit of the thermodynamic compressibility $\chi_{c} \rightarrow n(1-n / 2) / T$, while the diffusion constant saturates to a constant [5]. In the low temperature limit, on the other hand, the compressibility is expected to saturate, at least within a naive picture. Hence in this limit the temperature dependence should be dictated by the diffusion constant.

The main result of the paper by Brown et. al. is that while the diffusion constant and compressibility on their own follow non trivial temperature dependencies in the intermediate temperature regime, the resistivity constructed from the two, remains linear with a constant slope oblivious to crossing of the Mott-Ioffe-Regel limit (see figure). This result is at odds with a dynamical mean field theory (DMFT) calculation, which does indicate a change of the slope. The experiment agrees with an exact calculation, which cannot however be performed below the MIR limit due to finite size limitations. Thus, the experiment provides new insights on bad metal behavior that could not be obtained from unbiased numerical calculations.

Another important piece of information can be gleaned from the behavior of the com-

pressibility. Somewhat surprisingly, the measured compressibility continues to grow, showing no sign of saturating with decreasing temperature down to the lowest temperature of $0.3 t$. One explanation for this behavior is that the strong interactions generate a large effective mass that would lead to saturation only at a lower temperature. Another possibility is that the growing charge susceptibility is the harbinger of an approaching instability toward phase separation or a stripe phase.

In conclusion, the paper by Brown et. al. nicely demonstrates the power of ultra-cold atomic experiments to provide new insights on long standing questions in condensed matter physics. Even more exciting breakthroughs can be achieved if the atomic systems could be cooled to even lower temperatures. Overcoming this challenge would allow to determine the true low energy limit of the bad metal behavior in the Hubbard model. A possible next step could be to expand the investigation to a broader regime of parameters, for example toward weak coupling, and observe how the bad metal behavior evolves to the expected Fermi liquid behavior at weak coupling.

\section{References}

[1] C. M. Varma, P. B. Littlewood, S. Schmitt-Rink, e. Abrahams and A. E. Ruckenstein, Phys. Rev. Lett. 63, 1996-1999 (1989).

[2] H. v. Löhneysen, A. Rosch, M. Vojta, Matthias, and P. Wölfle, Rev. Mod. Phys. 79, 1015-1075 (2007).

[3] O. Parcollet and A. Georges, Phys. Rev. B 59, 5341 (1999).

[4] D. Chowdhury, Y. Werman, E. Berg, and T. Senthil, Phys. Rev. X 8, 031024 (2018)

[5] S. Mukerjee, V. Oganesyan and D. Huse, David, Phys. Rev. B 73, 035113 (2006) 